\title{
Stimulation of in vitro ovulation and contraction of brook trout (Salvelinus fontinalis) follicles by adrenaline through $\boldsymbol{\alpha}$-adrenoreceptors
}

\author{
F. W. Goetz and J. A. Bradley \\ University of Notre Dame, Department of Biological Sciences, Notre Dame, ID 46556, USA
}

\begin{abstract}
The effects of adrenaline and adrenoreceptor antagonists on ovulation and follicle wall contraction were investigated in brook trout (Salvelinus fontinalis) follicles using in vitro incubation systems. Adrenaline significantly stimulated a dose-dependent increase in ovulation and follicle contraction at concentrations between 1.0 and $100 \mu \mathrm{mol} \mathrm{I}^{-1}$. The ovulatory and contractile effects of $10 \mu \mathrm{mol}$ adrenaline $\mathrm{I}^{-1}$ could be blocked by the $\alpha_{1}$-adrenoreceptor antagonists WB-4101 and benoxathian, and by the $\alpha_{2}$-antagonist yohimbine. WB-4101 was the most potent blocker, significantly inhibiting ovulation and contraction at $1.0 \mu \mathrm{mol} \mathrm{l}^{-1}$. In contrast, the $\beta$-antagonist propranolol $\left(100-0.001 \mu \mathrm{mol} \mathrm{l}^{-1}\right)$ was totally ineffective in blocking adrenaline-induced ovulation and follicle contraction. The results indicate that there is a strong correlation between the effects of adrenaline on ovulation and contraction. In addition, the antagonist studies indicate that adrenaline stimulates ovulation and follicle contraction of brook trout follicles through $\alpha$-adrenoreceptors.
\end{abstract}

\section{Introduction}

Evidence that catecholamines play a direct role in the control of vertebrate ovulation comes from a variety of investigations (Goetz et al., 1991). However, the most convincing support comes from studies using ovaries perfused in vitro or incubation of isolated follicles, since the results of these studies cannot be attributed to extraovarian effects. It has been shown, for example, that hCG-induced ovulation in the perfused rabbit ovary can be partially blocked by antagonists of $\alpha$-adrenoreceptors and that noradrenaline can stimulate ovulation alone in the perfused rabbit ovary (Kobayashi et al., 1983; Schmidt et al., 1985) and the rat (Jorgensen et al., 1991) ovary. A review on the control of fish ovulation also indicated that adrenaline stimulates ovulation in isolated rainbow trout follicles through $\alpha$-adrenoreceptors (Jalabert, 1976). However, no data were actually shown in the paper.

It has been generally assumed that the way in which catecholamines influence ovulation is through the stimulation of smooth muscle contraction in the follicle wall. This contention is based on the results of earlier investigations showing that catecholamines can induce contraction of follicle wall strips (Walles et al., 1975, 1982). Further, noradrenaline-induced contraction is also blocked by $a$-adrenoreceptor antagonists (Walles et al., 1975, 1982). However, follicle contraction studies have always been conducted on the follicles of larger mammals such as cows, sheep and humans, whereas the ovulatory effects of catecholamine agonists and antagonists have been investigated on smaller animals such as rabbits and rats. Thus, whether catecholamines can stimulate ovulation in larger mammals and whether catecholamines stimulate contraction of

Received 20 March 1993 follicles in smaller mammals has not been reported. In addition, while the perfused ovary system may be free of extraovarian influences, it is still possible that the effects of certain agents in this system act through vascular changes in the follicle rather than directly on the follicle wall. Finally, while catecholamineinduced contraction does appear to be mediated by $\alpha$-adrenoreceptors (Walles et al., 1975; Owman et al., 1975), it is not at all clear which type of adrenoreceptors catecholamines are acting through in the stimulation of ovulation in perfused ovarian systems (Kobayashi et al., 1983; Schmidt et al., 1985).

Isolated trout follicles will ovulate in vitro if they have first undergone germinal vesicle breakdown (resumption of meiosis) in vivo (Goetz et al., 1982). In addition, we have recently developed an indirect in vitro method to study the contraction of trout follicles under agonist stimulation ( $\mathrm{Hsu}$ and Goetz, $1992 \mathrm{a}, \mathrm{b}$ ). Thus, using these in vitro methods we investigated the effects of catecholamines and their antagonists on ovulation and follicle contraction, in the same animal. Furthermore, given the very large number of follicles in individual trout, extensive dose responses with these agents could be recorded so that their activities could be compared. Our results show that adrenaline can stimulate both ovulation and contraction of brook trout follicles at similar concentrations, and in both cases the stimulation occurs through $\alpha$-adrenoreceptors.

\section{Materials and Methods}

\section{Animals and treatments}

Female brook trout were purchased from a private hatchery during the reproductive season (September) and held in flowthrough tanks at $12.5^{\circ} \mathrm{C}$ under natural photoperiods. Follicles 
were sampled from females as described by Goetz et al. (1982) to obtain fish in the proper maturational stage. For experiments, fish were anaesthetized in 2-phenoxyethanol and then decapitated. The ovaries were removed and dissected in ice-cold Cortland medium (Wolf and Quimby, 1969), containing $20 \mathrm{mmol} \mathrm{Hepes} \mathrm{l}^{-1}$ and adjusted to $\mathrm{pH} 7.8$ with $1.0 \mathrm{~mol}$ $\mathrm{NaOH} \mathrm{I}{ }^{-1}$. The mature follicles (approximately $4 \mathrm{~mm}$ diameter) were dissected from the extrafollicular tissue and immature follicles under a dissecting microscope. Only isolated follicles were used for incubations.

\section{Ovulation studies}

For ovulation studies, ovaries were removed from females following germinal vesicle breakdown and replicates of ten isolated follicles were incubated in $3.0 \mathrm{ml}$ medium (as above) in $25 \mathrm{ml}$ Erlenmeyer flasks at $12^{\circ} \mathrm{C}$ for various times depending on the fish and the rate of ovulation (specific times for each experiment are indicated in the legends to Figs 1-5). The agonist adrenaline bitartrate, and the antagonists WB-4101 hydrochloride (2-(2,6-dimethoxyphenoxy-ethyl)aminomethyl1,4-benzu-dioxane hydrochloride), benoxathian hydrochloride (2-[[[2-(2,6-dimethoxyphenoxy)ethyl]-amino]-methyl]-1,4-benoxathian hydrochloride), yohimbine hydrochloride (17hydroxyyohimban-16-carboxylic acid methyl ester hydrochloride), and propranolol hydrochloride were dissolved directly in the medium. Adrenaline and propranolol were purchased from Sigma (St Louis, MD) while WB-4101, benoxathian and yohimbine were purchased from Research Biochemical Inc. (Natick, MA). After $48 \mathrm{~h}$ of incubation, or when the amount of ovulation reached a plateau, ovulation was quantified by counting the number of spent follicles in the incubates. This number was then expressed as a percentage of the total number of follicles per flask.

\section{Follicle contraction}

The assay of follicle contraction was conducted as described by Hsu and Goetz (1992a, b). Briefly, isolated follicles were dissected as described above, and were then punctured once with a 25-gauge hypodermic needle. The puncture extended completely through the follicle wall and the egg membrane. Thus, if the follicle wall contracted when treated with an agonist, yolk would be expelled through the puncture and into the medium, resulting in a decrease in the weight of the follicle. The greater the contractile force, the larger the weight loss. Punctured follicles were incubated as described above for the ovulation assay. Replicates of ten follicles were incubated in $3.0 \mathrm{ml}$ of medium in $25 \mathrm{ml}$ Erlenmeyer flasks for $16 \mathrm{~h}$ at $12^{\circ} \mathrm{C}$. At the end of incubation, the follicles in each replicate were collected in a small preweighed plastic dish and the medium surrounding them was removed by a Pasteur pipette and Kimwipes. The follicles were then weighed to the nearest $0.1 \mathrm{mg}$. The contractility in agonist incubates was then expressed as a percentage of the weight of unpunctured follicle controls. Untreated, punctured controls were always run for comparison to determine the amount of agonist-induced versus spontaneous contraction.
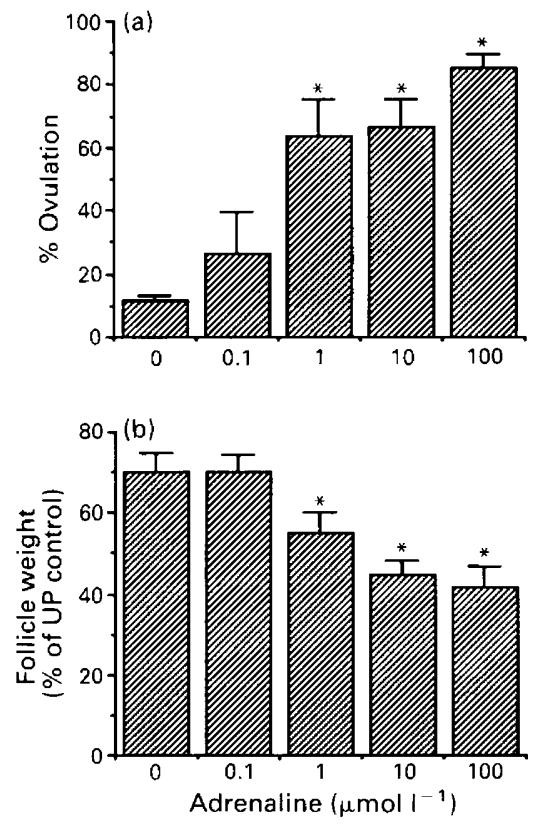

Fig. 1. Effects of adrenaline on (a) ovulation and (b) contraction [calculated as the percentage of the follicle weight of unpunctured (UP) controls] of brook trout follicles. Each bar is the mean of experiments conducted on the follicles of four fish for ovulation and five fish for contraction. Within the experiments on each fish, each treatment was conducted in duplicate. All four fish in the ovulation experiment were assayed following $12 \mathrm{~h}$ of incubation. *Significantly different from incubates without adrenaline $(P<0.05)$.

In contraction experiments, follicles were obtained from fish before germinal vesicle breakdown rather than just before ovulation. This is because ovaries are much easier to obtain before germinal vesicle breakdown and the preovulatory ovaries were needed for testing the effects of agonists and antagonists on ovulation. In addition, preliminary experiments indicated that follicles before germinal vesicle breakdown responded to adrenaline and the response was similar to preovulatory follicles.

\section{Statistical analysis}

Ovulation and follicle contraction data were transformed using the arcsine transformation before statistical analysis. Statistical significance was assessed by ANOVA followed by Duncan's multiple range test. $P$ values less than 0.05 were considered significant.

\section{Results}

Ovulation was significantly stimulated in isolated follicles by adrenaline at concentrations between 1.0 and $100 \mu \mathrm{mol} \mathrm{l}^{-1}$ (Fig. 1). The same adrenaline concentrations also significantly stimulated follicle contraction when compared with untreated, unpunctured controls (Fig. 1). As observed in previous reports (Hsu and Goetz, 1992a, b), there was a 30\% spontaneous (in the absence of agonists) weight loss in punctured controls (i.e. $0.0 \mu \mathrm{mol}$ adrenaline $\mathrm{I}^{-1}$ ). 

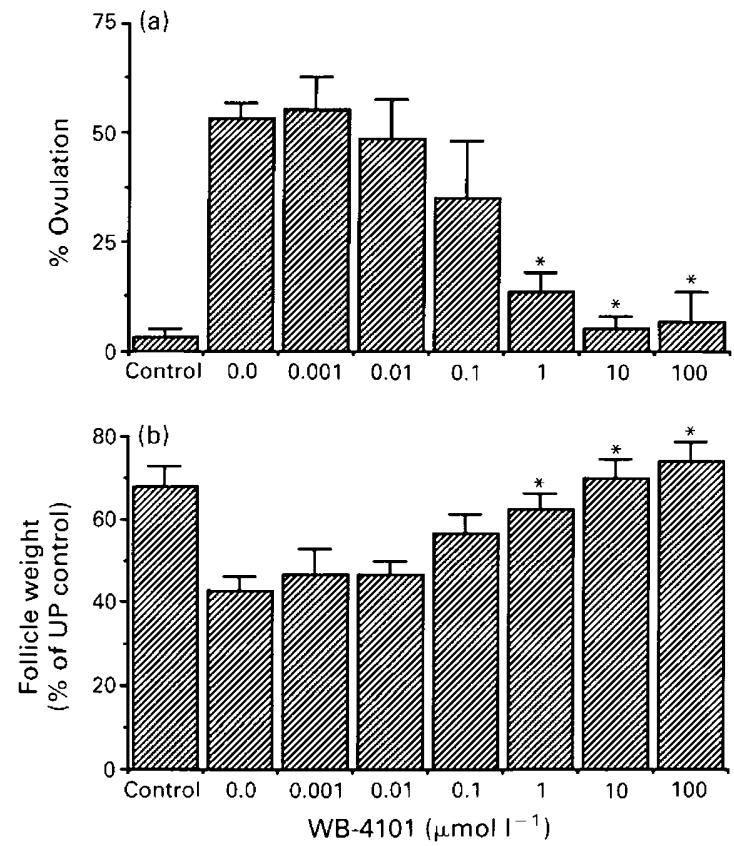

Fig. 2. Effects of WB-4IOI on (a) ovulation and (b) contraction induced by $10 \mu \mathrm{mol}$ adrenaline $\mathrm{I}^{-1}$ (calculated as the percentage of the follicle weight of unpunctured controls). Each bar is the mean of experiments conducted on the follicles of three fish. Within the experiments on each fish, each treatment was conducted in duplicate. In the ovulation experiment, two of the fish were assayed at $40 \mathrm{~h}$ of incubation and the other at $12 \mathrm{~h}$. *Significantly different from adrenaline incubates without WB-4101 at $P<0.05$.

The effects of adrenoreceptor antagonists were tested against the ovulatory and contractile effects of adrenaline at a fixed concentration of $10 \mu \mathrm{mol} \mathrm{I}^{-1}$. While $1.0 \mu \mathrm{mol}$ adrenaline $1^{-1}$ also significantly stimulated both ovulation and contraction, a concentration of $10 \mu \mathrm{mol} \mathrm{l^{-1 }}$ was chosen since this concentration stimulated the same amount of ovulation but a greater amount of contraction. At concentrations between 1.0 and $100 \mu \mathrm{mol} \mathrm{l}^{-1}$, WB-410I significantly blocked the effects of $10 \mu \mathrm{mol}$ adrenaline $\mathrm{l}^{-1}$ on ovulation and contraction (Fig. 2). Benoxathian also significantly blocked the contractile effect of adrenaline but was approximately 10 times less potent than WB-4101 (Fig. 3). As with WB-4101, there was also a dose-related inhibition of ovulation by benoxathian, but this was only significant at $100 \mu \mathrm{mol} 1^{-1}$.

At 10 and $100 \mu \mathrm{mol} \mathrm{l}^{-1}$, yohimbine significantly blocked contraction induced by $10 \mu \mathrm{mol}$ adrenaline $\mathrm{l}^{-1}$ (Fig. 4). This antagonist also significantly blocked ovulation at 10 and $100 \mu \mathrm{mol} \mathrm{l}^{-1}$ but appeared to exhibit a slightly different effect on ovulation at lower concentrations. At a concentration of $0.001 \mu \mathrm{mol} \mathrm{I}^{-1}$, yohimbine appeared to increase ovulation above that observed with $10 \mu \mathrm{mol}$ adrenaline $\mathrm{I}^{-1}$ alone; however, this was not significantly different.

Propranolol did not have a significant effect on contraction or ovulation at concentrations between 0.001 and $100 \mu \mathrm{mol}$ $1^{-1}$ (Fig. 5).

The ovilatory and contractile effects of each of the antagonists alone at 1 and $100 \mu \mathrm{mol} \mathrm{l^{-1 }}$ are shown (Table 1 ). When tested alone, none of the antagonists had any significant effect on contraction when compared with punctured con-
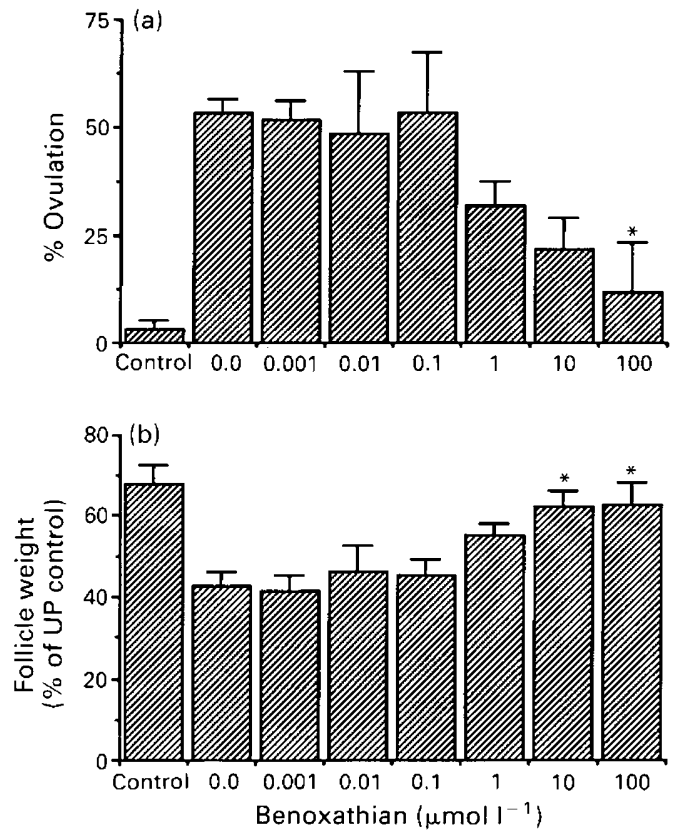

Fig. 3. Effects of benoxathian on (a) ovulation and (b) contraction induced by $10 \mu \mathrm{mol}$ adrenaline $1^{-1}$ (calculated as the percentage of the follicle weight of unpunctured controls). Each bar is the mean of experiments conducted on the follicles of three fish. Within the experiments on each fish, each treatment was conducted in duplicate. In the ovulation experiment, two of the fish were assayed at $40 \mathrm{~h}$ of incubation and the other at $12 \mathrm{~h}$. ${ }^{*}$ Significantly different from adrenaline incubates without benoxathian $(P<0.05)$.

trols, or ovulation when compared with controls without adrenaline.

\section{Discussion}

The resuits of this study clearly demonstrate that adrenaline can stimulate both ovulation and contraction of brook trout follicles at levels that are comparable to those previously used in mammalian investigations. Further, these effects could be blocked by $\alpha$-adrenergic antagonists but not by a $\beta$-antagonist. While previous studies have shown that catecholamines can stimulate ovulation in fish and mammals (Jalabert, 1976; Kobayashi et al., 1983; Schmidt et al., 1985; Jorgensen et al., 1991), the results presented here are significant for several reasons. First, while adrenaline and noradrenaline have been reported to stimulate contraction of follicle walls (Walles et al., 1975; Owman et al., 1975), contraction studies have only been conducted on the follicles of large mammals in which the ovulatory effects of catecholamines have not been investigated. In contrast, in those species in which the ovulatory effects of catecholamine agonists and antagonists have been studied, there have been no investigations on the effects of these agents on follicle contractility. The results presented here conclusively demonstrate that both processes are stimulated in the follicles from a single vertebrate, the brook trout, and that there is a correlation between the doses required to stimulate these two processes by catecholamines. Furthermore, both assays were 

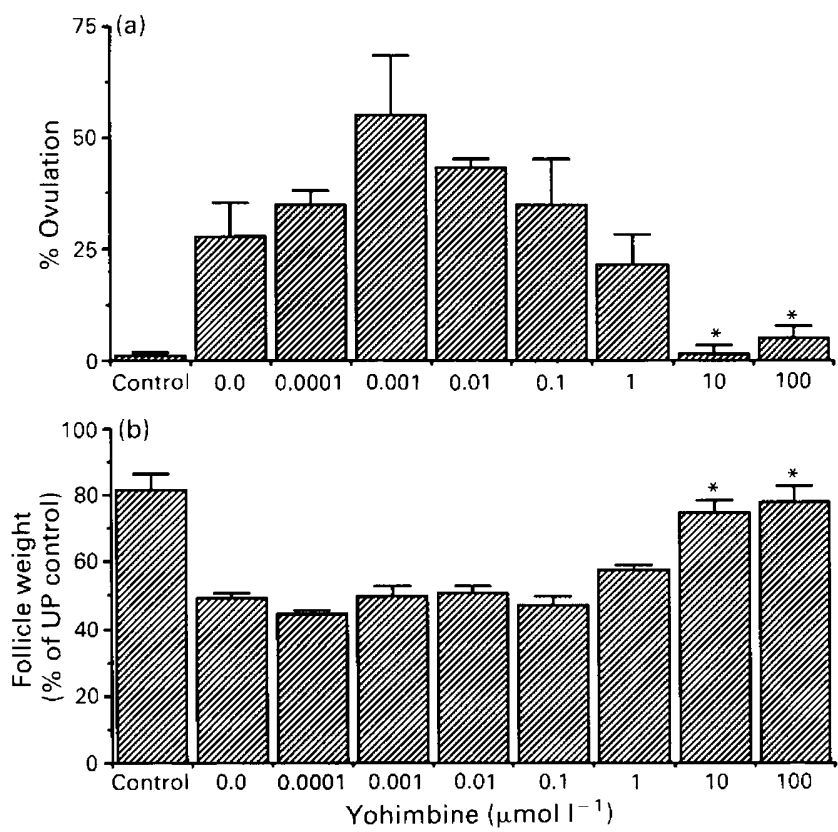

Fig. 4. Effects of yohimbine on (a) ovulation and (b) contraction induced by $10 \mu \mathrm{mol}$ adrenaline $1^{-1}$ (calculated as the percentage of the follicle weight of unpunctured controls). Each bar is the mean of experiments conducted on the follicles of three fish. Within the experiments on each fish, each treatment was conducted in duplicate. In the ovulation experiments, two of the fish were assayed at $48 \mathrm{~h}$ and the other at $30 \mathrm{~h}$. ${ }^{*}$ Significantly different from adrenaline incubates without yohimbine $(P<0.05)$.

conducted completely in vitro without perfusion through the vasculature, indicating that the effects are directly on the follicle wall.

In the few studies that have looked at the (ovarian perfusion) effects of catecholamines on ovulation in vitro, it is not clear which adrenergic pathway is being stimulated. In rabbits, neither phenoxybenzamine nor phentolamine could block the ovulatory effects of noradrenaline (Kobayashi et al., 1983); however, $\beta$-antagonists were not tested for comparison. It does appear that gonadotrophin-induced ovulation in the perfused rabbit ovary involves $\alpha$-adrenergic stimulation, since this can be blocked (though not totally) with phenoxybenzamine and phentolamine (Kobayashi et al., 1983; Schmidt et al., 1985). There have been a number of investigations on the effects of catecholamines on follicle contraction primarily using follicle wall strips from bovine and human ovarian follicles. The results consistently indicate that in these vertebrates noradrenaline is slightly more potent than adrenaline in stimulating contraction, and that $\alpha$ antagonists can block this stimulation (Walles et al., 1975; Owman et al., 1975). Thus, this data support the contention that the effects of catecholamines on ovulation are mediated by $\alpha$-adrenergic-mediated follicle wall contraction.

While a few studies on follicle wall contraction have used receptor subtype specific $\alpha$ antagonists, investigations on ovulation have used only general $\alpha$ antagonists. In the present investigation, we used antagonists that are reported to have very different effects on the $\alpha_{1}$ - and $\alpha_{2}$-adrenoreceptor subtypes. WB-4101 and benoxathian are very similar compounds and have high selectivity for the $\alpha_{1}$ subtype over $\alpha_{2}$ receptors
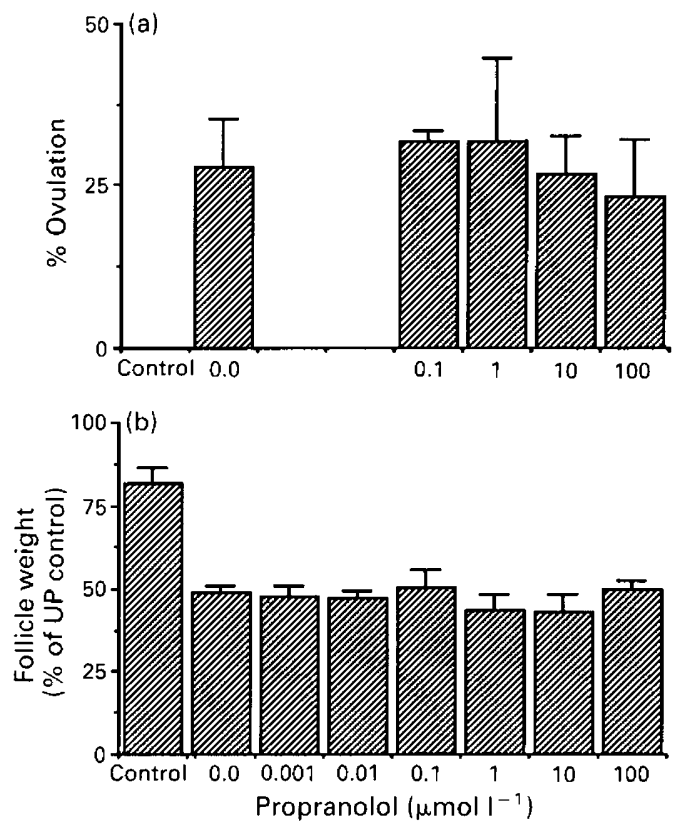

Fig. 5. Effect of propranolol on (a) ovulation and (b) contraction induced by $10 \mu \mathrm{mol}$ adrenaline $\mathrm{l}^{-1}$ (calculated as the percentage of the follicle weight of unpunctured controls). Each bar is the mean of experiments conducted on the follicles of three fish. Within the experiments on each fish, each treatment was conducted in duplicate. In the ovulation experiments, two of the fish were assayed at $48 \mathrm{~h}$ and the other at $30 \mathrm{~h}$. In one of the three fish, the ovulatory effects of propranolol were investigated only at concentrations between 0.1 and $100 \mu \mathrm{mol} \mathrm{l}^{-1}$, and so only the data for these treatments are shown. No significant effects were observed.

Table 1. Effects of $\alpha$ - and $\beta$-adrenoreceptor antagonists on brook trout follicle contraction and ovulation in the absence of adrenaline

\begin{tabular}{lrcc}
\hline Antagonist & $\begin{array}{c}\text { Concentration } \\
\left.(\mu \mathrm{mol} \mathrm{l})^{-1}\right)\end{array}$ & $\begin{array}{c}\text { Follicle weight } \\
(\%)\end{array}$ & $\begin{array}{c}\text { Ovulation } \\
(\%)\end{array}$ \\
\hline Propranolol & 100.0 & $77.4 \pm 5.3^{\mathrm{a}}$ & $0.0^{\mathrm{b}}$ \\
& 1.0 & $80.9 \pm 1.9$ & 0.0 \\
Yohimbine & 0.0 & $81.8 \pm 4.4$ & 0.0 \\
& 100.0 & $75.4 \pm 4.1$ & $3.3 \pm 1.7$ \\
WB-4101 & 1.0 & $78.6 \pm 4.0$ & 0.0 \\
& 0.0 & $81.8 \pm 4.4$ & 0.0 \\
Benoxathian & 100.0 & $67.7 \pm 8.3$ & $10.0 \pm 5.0$ \\
& 1.0 & $69.3 \pm 8.1$ & $5.0 \pm 2.9$ \\
& 0.0 & $68.2 \pm 3.8$ & $3.3 \pm 2.0$ \\
& 100.0 & $62.5 \pm 7.2$ & $5.0 \pm 2.9$ \\
& 1.0 & $68.3 \pm 6.6$ & $3.3 \pm 1.7$ \\
& 0.0 & $68.2 \pm 3.8$ & $3.3 \pm 2.0$
\end{tabular}

avalues are means ( \pm SEM) follicle weights, calculated as the percentage of unpunctured controls, for experiments conducted on the follicles of three fish, each treatment performed in replicate per fish.

${ }^{b}$ Mean ( \pm SEM) percentage ovulation from experiments conducted on three fish, each treatment performed in replicate per fish.

(Pigini et al., 1988). In contrast, yohimbine is an $\alpha_{2}$ adrenoreceptor antagonist. In the experiments described here, 
the most potent antagonist was WB-4101. This antagonist could block the effects of adrenaline at $0.1 \mu \mathrm{mol}^{-1}$. However, yohimbine could still block contraction and ovulation, and the results for this antagonist were not very different from another $\alpha_{1}$ antagonist, benoxathian. Thus, at this point it is not possible to determine which $\alpha$-adrenoreceptor adrenaline acts through. It is clear, however, that $\beta$-adrenoreceptors are not involved at least in the stimulatory effects of adrenaline on ovulation and contraction - since propranolol could not block adrenalineinduced contraction or ovulation. Furthermore, it appeared that the ovulatory response to yohimbine may have actually been different from that with the $\alpha_{1}$ antagonists. At very low concentrations, yohimbine appeared to enhance the stimulatory effect of adrenaline, although the effect was not significant. It is possible that at higher concentrations, yohimbine was actually blocking all $\alpha$-adrenoreceptors, whereas at lower concentrations it may have selectively blocked $\alpha_{2}$-adrenoreceptors. It is clear that yohimbine did not enhance contraction at lower concentrations.

As shown in previous studies, there was a decrease in the weight of punctured follicles even in the absence of agonists or antagonists. This spontaneous loss of yolk is probably an active process requiring contraction, as we have shown (Hsu and Goetz, 1992b) that it can be inhibited by certain agents, such as forskolin and E prostaglandins, that increase the concentration of cAMP. While the adrenoreceptor antagonists had effects on adrenaline-stimulated contraction, they did not have any effects of their own on the spontaneous contraction exhibited by punctured controls. Thus, it appears that catecholamines are not responsible for the spontaneous contraction observed in the follicle before the germinal vesicle breakdown stage. In preliminary experiments using preovulatory follicles, we have observed that the amount of spontaneous contraction is higher than that observed with follicles before germinal vesicle breakdown and that the adrenaline effect is also greater. It is possible that this enhanced spontaneous contraction is a result of catecholamine action and this will have to be investigated.

Finally, the fact that adrenaline could stimulate contraction of follicles before germinal vesicle breakdown indicates that the receptors and the ability to contract are present in the follicle before ovulation. Oocytes before germinal vesicle breakdown will not ovulate when incubated in vitro with adrenaline or with other ovulatory agents such as prostaglandins. In fact, trout oocytes will not ovulate in vitro unless they have already undergone germinal vesicle breakdown in vivo (Goetz et al, 1982). Thus, other preparatory processes, such as proteolysis, must be necessary before adrenaline can stimulate ovulation.

Supported by NIH Research Grant HD25924-02.

\section{References}

Goetz FW, Smith DC and Krickl SP (1982) The effects of prostaglandins, phosphodiesterase inhibitors and cyclic AMP on ovulation of brook trout (Salvelinus fontinalis) oocytes General and Comparative Endocrinology 48 $154-160$

Goetz FW, Berndtson A and Ranjan M (1991) Ovulation: Mediators at the ovarian level Vertebrate Endocrinology, Fundamentals and Biomedical Implications Vol. IV (A) Reproduction pp 127-203 Eds P Pang and M Schreibman. Academic Press, New York

Hsu T and Goetz FW (1992a) Angiotensins stimulate in vitro ovulation and contraction of brook trout (Salvelinus fontinalis) follicles Fish Physiology and Biochemistry 10 277-282

Hsu T and Goetz FW (1992b) The effects of E and F prostaglandins on ovarian cAMP production and follicular contraction in the brook trout (Salvelinus fontinalis) General and Comparative Endocrinology 88 434-443

Jalabert B (1976) In vitro oocyte maturation and ovulation in rainbow trout (Salmo gairdneri), northern pike (Esox lucius), and goldfish (Carassius auratus) Journal of the Fisheries Research Board of Canada 33 974-988

Jorgensen JCh, Kannistro P, Liedberg F, Ottesen B, Owman C and Schmidt G (1991) The influence of neuropeptide $Y$ and norepinephrine on ovulation in the rat ovary Peptides 12 975-982

Kobayashi Y, Sjoberg ND, Walles B, Owman Ch, Wright KH, Santulli R and Wallach EE (1983) The effect of adrenergic agents on the ovulatory process in the in vitro perfused rabbit ovary American Journal of Obstetrics and Gynecology 145 857-864

Owman Ch, Sjoberg N-O, Svensson KG and Walles B (1975) Autonomic nerves mediating contractility in the human graafian follicle Journal of Reproduction and Fertility 45 553-556

Pigini M, Brasili L, Giannella M, Giardina D, Gulini U, Quaglia W and Melchiorre C (1988) Structure-activity relationships in 1,4-Benzodioxan-related compounds. Investigation on the role of the dehydrodioxane ring on $\alpha$-adrenoreceptor blocking activity Journal of Medicinal Chemistry 312300 2304

Schmidt G, Owman Ch, Sjoberg N-O and Walles B (1985) Influence of adrenoreceptor agonists and antagonists on ovulation in the rabbit ovary perfused in vitro Joumal of Autonomic Pharmacology 5 241-250

Walles B, Edvinsson L, Owman C, Sjoberg N-O and Svensson KG (1975) Mechanical response in the wall of ovarian follicles mediated by adrenergic receptors Journal of Pharmacology and Experimental Therapeutics 193 460-473

Walles B, Owman Ch and Sjoberg N-O (1982) Contraction of the ovarian follicle induced by local stimulation of its sympathetic nerves Brain Research Bulletin $9757-760$

Wolf K and Quimby MC (1969) Fish cell and tissue culture Fish Physiology. Vol. 3 pp 253-305 Eds WS Hoar and DJ Randall. Academic Press, New York. 